\title{
Augmented Learning for Sports Using Wearable Head-worn and Wrist-worn Devices
}

\author{
Hui-Shyong Yeo* \\ University of St Andrews
}

\author{
Hideki Koike ${ }^{\dagger}$ \\ Tokyo Institute of Technology
}

\author{
Aaron Quigley \\ University of St Andrews
}

\begin{abstract}
Novices can learn sports in a variety of ways ranging from guidance from an instructor to watching video tutorials. In each case, subsequent and repeated self-directed practice sessions are an essential step. However, during such self-directed practice, constant guidance and feedback is absent. As a result, the novices do not know if they are making mistake or if there are any areas for improvement. In this position paper, we propose using wearable devices to augment such self-directed practice sessions by providing augmented guidance and feedback. In particular, a head-worn display can provide real-time guidance whilst wrist-worn devices can provide real-time tracking and monitoring of various states. We envision this approach being applied to various sports, and in particular this is suitable for sports that utilize precise hand motion such as snooker, billiards, golf, archery, cricket, tennis and table tennis
\end{abstract}

Index Terms: H.5.2 [User Interfaces]: User Interfaces-Graphical user interfaces (GUI); H.5.m [Information Interfaces and Presentation]: Miscellaneous

\section{INTRODUCTION}

How do novices acquire sports skill from an expert? Such novices could either attend class and learn from instructors, or their learning style might better suit learning from watching video tutorials. In either case, often the key to success is to have a lot of practice.

However, novices practicing by themselves often struggle with problems. For example, they would not know if they are performing particular movements correctly or if they are making serious mistakes, in addition they won't know how to avoid such mistakes and improve their technique. Unfortunately, it would be very costly to hire and practice with the instructor (expert) all the time. Therefore, there is a need for a system that can support novices practicing on their own effectively and efficiently. Such a system should provide constant guidance and feedback on the user's skill, including posture, motion and action. Skill acquisition mechanisms and a skill transfer systems [7] may further enhance the learning opportunities for novice users.

Besides just real-time guidance based on computer systems, such a system could also allow remote telepresence training session with real human instructors. In addition, it could collect a database of recorded training data from expert athletes, and could, for example, transfer to the novices using a system such as artificial muscle or movements with an exoskeleton.

To tackle this research problem, we propose a framework that utilizes commodity wearable devices such as head-mounted display and smartwatch, for both displaying the guidance and for capturing and correcting novices posture and action in real-time. This system could be adapted to most of the sports that involve wrist motion,

\footnotetext{
*e-mail: hsy@st-andrews.ac.uk

†e-mail: koike@c.titech.ac.jp

†e-mail: aquigley@st-andrews.ac.uk
}

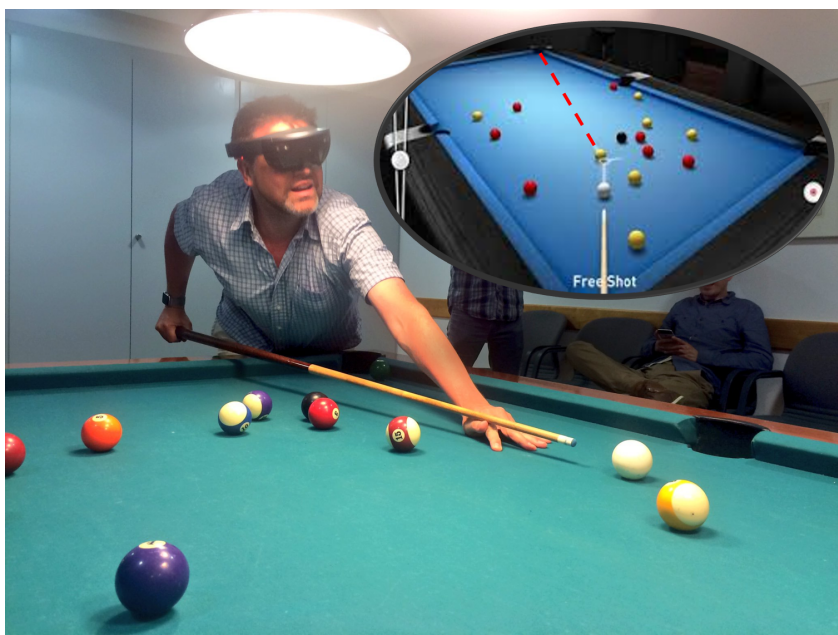

Figure 1: Envisioned augmented snooker by wearing HoloLens where the inset figure shows guidelines and estimated path.

such as swing-based ball sports (tennis, table tennis, golf, baseball, billiards) or other type of sports (throwing disk, archery or kayaking).

\section{Motivation}

The motivation to approach this problem with such a platform are two-fold. Commodity wearable devices such as smartwatches and fitness bands are low-cost, ubiquitous and accessible, where many people are already wearing them. This allows capturing of performance easily, anytime and in-the-wild, without requiring experts to come into a lab for data capture, nor requires complex setup of equipment outdoor, not to mention that typical motion capture system (Vicon) might not work under sunlight. In addition, the requirement of using complicated sensors for training can be expensive, cumbersome and possess a high barrier of entry for novice users to even start using the system.

\section{Related Work}

\subsection{Academic Research}

Tanaka et al. [12] (Figure 2) have proposed and implemented a similar system for real-time golf-swing training system using sonification and sound image localization. However, the system is based on sound rather than visual feedback, hence it does not provide visual information such as the expected trajectory or the current wind speed. In addition, the hardware relies on mounting 3D tracker markers on the head and the golf club, which can affect the golf-swing.

There are various projection-based Augmented Reality (AR) tables for Billiard that show projected ball trajectory. This type of system requires permanent setup of projector-camera system. It is also difficult to estimate the angle of the cue due to occlusion.

In PingPongPlus [6], Ishii et al. proposed a novel interface for digitally-augmented cooperative play and the concept of the athletictangible interface. By contrast, Blank et al. [2] present a sensor- 

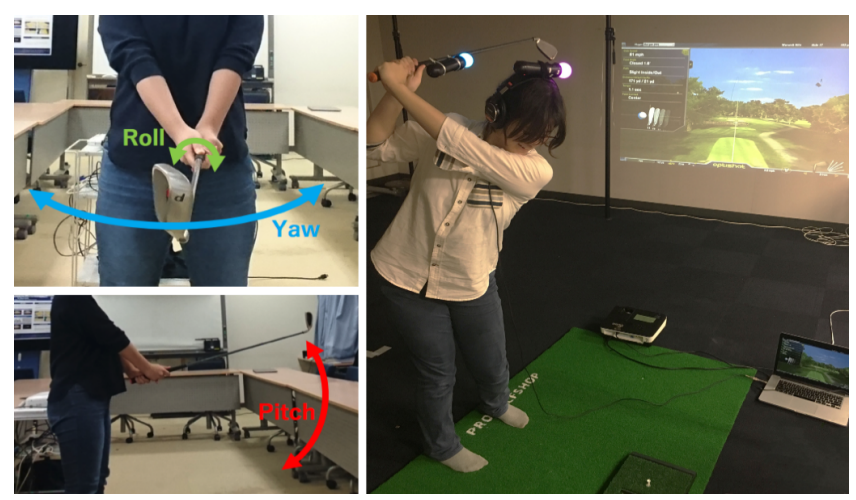

Figure 2: Real-time golf-swing training system using sonification and sound image localization from Tanaka et al. [12]

based stroke detection and stroke type classification in table tennis. Subsequent work [1] presented an approach for ball speed and spin estimation based on a single racket-mounted inertial sensor.

Gowda et al. [3,4] presented an IoT platform for sports analytics. It relies on embedded inertial measurement unit and RFID chip inside cricket balls. On the other hand, MV-Sports [13] is a costeffective system for real-time sports analysis based on motion and vision sensor integration. Taking tennis as a case study, it aims to recognize player shot types and measure ball states. Wearable devices have also been used in supporting the learning of climbing by providing the actors involved with augmented communication [9].

A smart soccer shoe [14] can monitor foot-ball interaction with integrated textile pressure sensor. Pai et al. [10] introduce the concept of ubiquitous sports training where the user can train anytime, anywhere using MR and VR. Gradl et al. [5] propose the use of these AR/VR systems in the field of sports by combining it with radio-based localization technology and found that majority people are inclined to use it after being confronted with possible usage scenarios.

\subsection{Commercial Products}

On the commercial side, there are various commercial products that aim to improve sports skill acquisition. For example, Babolat ${ }^{1}$ and Sony ${ }^{2}$ present the tennis player an overview of the game by analyzing data collected from sensors in the racket handle, although the latter was discontinued due to deployment problems. K-VEST ${ }^{3}$ is a human motion learning system to analyze and teach golf-swing in real-time. In addition, eye-tracking ${ }^{4}$ technology can be used to enhance badminton player performance.

There are also various VR and non-VR sport games such as those available on PlayStation and Oculus platform. These systems can also be used to practice and improve sports skill, although it might lack realism due to lack of haptic and tactile feedback. Nonetheless, such systems with a built-in Artificial Intelligence (AI) system may allow the novice to improve their practice sessions.

\section{DEsign AND Implementation}

In this section we will describe our envisioned framework design and architecture, including hardware and software requirements. Here we could apply machine learning and deep learning technique to train the data from the wearable devices by using ground truth data from the motion capture system. Later we can compare the performance against the expert athletes data stored in database.

\footnotetext{
${ }^{1}$ http://en.babolatplay.com/

${ }^{2} \mathrm{http} / / / \mathrm{www}$. smarttennissensor.sony.net/

${ }^{3}$ https://www.k-motion.com/

${ }^{4}$ https://www.youtube.com/watch?v=0W7FqDBD7Ts
}

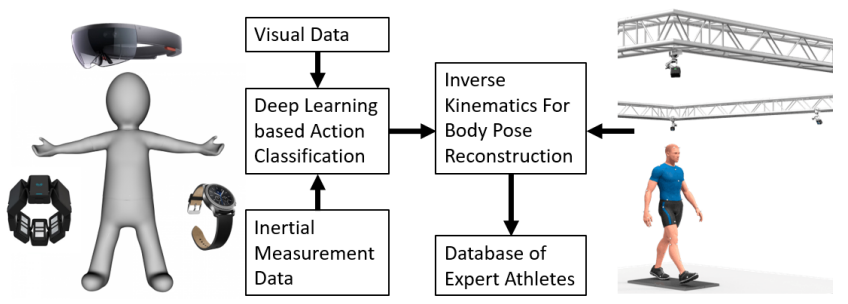

Figure 3: Framework architecture, left is motion and visual data while right is ground truth data used for training.

\subsection{Hardware Details}

Smartwatches are now equipped with various sensors, including high speed IMU (accelerometer, gyroscope and magnetometer), heart rate sensor, camera, microphone, speaker etc. These sensors can capture useful data. For example, the IMU running at high speed $(4 \mathrm{kHz}$ accelerometer and $32 \mathrm{kHz}$ gyroscope) can capture high speed motion or hand action [8]. The camera can also capture hand pose [11] or real-world environment, which might be useful for SLAM-based tracking. Microphone and speaker can be useful for acoustic-based sensing (e.g., sonar, Doppler). While a heart rate sensor can be useful to keep track of the users optimal state.

Indeed, many smartwatches and fitness trackers on the market can already track different kind of sports activity. However, they are often limited to just tracking basic information such as the calories burned, repetitions and steps. The goal in this project is different from just capturing such basic information, but rather focuses on capturing the high fidelity information such as wrist motion, swing angle and swing speed, and hence combining this information for real-time visual feedback.

Our proposed platform aims to be extensible as there are many other off-the-shelf devices which we might incorporate. Other such devices include, wearables such as Myo and Ctrl-Lab EMG armband, Mudra wrist band, Jins Meme and Pupil Labs eye tracker, Mbient Lab Meta IMUs and Microsoft Band Fitness activity tracker. Technology shown in academic research projects such as Tomo arm band, WristFlex and ViBand can also be adopted. Another type of sensor to consider is low-cost radar sensor such as the Google Project Soli or Walabot, which can provide wide field-of-view and high speed measurement of motion. For real-time augmented visualizations we are considering Magic Leap or Microsoft HoloLens.

Our goal is not to instrument the users with infrared markers or require the users to wear motion capture suit that can only be captured in complex lab environments. We aim to be as portable as possible, requiring the user to only wear off-the-shelf wearable devices such as smartwatches, wrist band or smartglass. Thus, the system should be able to deploy in the wild and real-world environment where sports normally take place instead of limited to controlled in-lab environments only.

However, in order to evaluate our proposed framework, we need to have a reliable system to provide ground truth data that can be used for comparison and evaluation. Such a system can be the Vicon or OptiTrack motion capture system or other low-cost alternative such as the HTC Vive Lighthouse system or Microsoft Kinect, although with higher latency and tracking error. These are only used during evaluation of our framework, but not during actual deployment.

\subsection{Software Details}

The sensor data from the wearable devices will be streamed to a host using either Bluetooth or WiFi technology. To reduce streaming latency we plan to employ a high end WiFi router that supports at least 802.11ac protocol. The proposed infrastructure can be seen in 
Figure 3 . We plan to use the Unity $3 \mathrm{D}$ game engine to implement the framework and TensorFlow for deep learning.

\section{Use Cases}

Here we suggest several potential use cases.

Billiards Let's consider Billiards as an example use case (Figure 1). A smartwatch could track the hand movement speed and direction, while a mixed reality headset could provide guidelines and information about expected ball trajectory (Figure 1 inset). Unlike existing projection mapping based tables, our system based on headset and smartwatch requires no infrastructure. It can map and track the scene in real-time, provide first-person view of visual guidance, tracks and records hand motion and speed with high fidelity.

Archery A second use case is archery ${ }^{5}$, which can be either VR or AR based. In a VR based system, it is convenient because no real arrows are required. In addition, virtual environment such as the effect of wind can be easily simulated, making the training more effective under different conditions. However it might lack realism, especially haptic and tactile feedback. In AR based system however, it is more realistic at the cost of using real arrows and a larger archery field for training. In both cases, the headset could display the projected trajectory depending on the string pull strength and angle of the bow. The system will make use of real-time wind speed and applying physics calculation to the projected arrow trajectory. As archery requires stability and concentration, the system could also measure the user condition such as heart rate and breath rate and alert the user to release the arrow when the user is calm.

Golf A third use case is with golf. The smartwatch could measure the body posture, angle and swing speed of the club. The system can alert the user about wrong body posture, club choice or grip position. Similar to archery, the system could also display the projected trajectory based on hit angle and suggest swing strength. Again it could make use of real-time wind speed and weather condition.

Table Tennis Stroke angle and stroke speed can be measured using high speed accelerometer and gyroscope built-into a smartwatch. Different type of spin (top spin, side spin) can be classified. Eye tracking (e.g., Magic Leap, Pupil Labs glass) technology can be use to track the user gaze and suggest improvement.

\section{FUtURE WORK}

Rather than limiting the potential of this framework to assist learning only, another research interest is on augmenting sports for recreational purposes. For example, the system could make the sport more challenging and fun by augmenting certain aspects of it such as introducing obstacle or inducing impairment by blocking the user field-of-view with virtual disruptors. This in essence turns a classical sport into a new interactive game. For example in table tennis, instead of trying to win, the goal is to hit certain area of the table to score points. Or users can cast spells which result in the addition of virtual distruptors to appear in their opponent's view.

\section{Conclusion ANd Limitation}

We have proposed a framework for augmenting sports learning by leveraging wearable devices. Over the next few months, we plan to develop the framework and conduct an evaluation.

Unlike motion capture system, wearable devices do not provide position tracking, which is a trade-off for portability. Another limitation is the limited battery capacity on these devices, which requires frequent charging. Nonetheless, we believe the benefits of such a portable system outweighs the trade-offs of a complex system.

\section{REFERENCES}

[1] P. Blank, B. H. Groh, and B. M. Eskofier. Ball speed and spin estimation in table tennis using a racket-mounted inertial sensor. In Proceedings of the 2017 ACM International Symposium on Wearable Computers, ISWC '17, pp. 2-9. ACM, New York, NY, USA, 2017. doi: 10.1145/ 3123021.3123040

[2] P. Blank, J. Hoßbach, D. Schuldhaus, and B. M. Eskofier. Sensorbased stroke detection and stroke type classification in table tennis. In Proceedings of the 2015 ACM International Symposium on Wearable Computers, ISWC '15, pp. 93-100. ACM, New York, NY, USA, 2015. doi: $10.1145 / 2802083.2802087$

[3] M. Gowda, A. Dhekne, S. Shen, R. R. Choudhury, L. Yang, S. Golwalkar, and A. Essanian. Bringing iot to sports analytics. In 14th USENIX Symposium on Networked Systems Design and Implementation (NSDI 17), pp. 499-513. USENIX Association, Boston, MA, 2017.

[4] M. Gowda, A. Dhekne, S. Shen, R. R. Choudhury, S. X. Yang, L. Yang, S. Golwalkar, and A. Essanian. Iot platform for sports analytics. GetMobile: Mobile Comp. and Comm., 21(4):8-14, Feb. 2018. doi: 10. 1145/3191789.3191793

[5] S. Gradl, B. M. Eskofier, D. Eskofier, C. Mutschler, and S. Otto. Virtual and augmented reality in sports: An overview and acceptance study. In Proceedings of the 2016 ACM International Joint Conference on Pervasive and Ubiquitous Computing: Adjunct, UbiComp '16, pp. 885-888. ACM, New York, NY, USA, 2016. doi: 10.1145/2968219. 2968572

[6] H. Ishii, C. Wisneski, J. Orbanes, B. Chun, and J. Paradiso. Pingpongplus: Design of an athletic-tangible interface for computer-supported cooperative play. In Proceedings of the SIGCHI Conference on Human Factors in Computing Systems, CHI '99, pp. 394-401. ACM, New York, NY, USA, 1999. doi: 10.1145/302979.303115

[7] H. Koike, J. Rekimoto, J. Ushiba, S. Furuya, and A. Ito. A study on skill acquisition mechanism and development of skill transfer systems. In CEUR Workshop Proceedings, vol. 2068, 2018.

[8] G. Laput, R. Xiao, and C. Harrison. Viband: High-fidelity bio-acoustic sensing using commodity smartwatch accelerometers. In Proceedings of the 29th Annual Symposium on User Interface Software and Technology, UIST '16, pp. 321-333. ACM, New York, NY, USA, 2016. doi: $10.1145 / 2984511.2984582$

[9] E. Mencarini, C. Leonardi, A. De Angeli, and M. Zancanaro. Design opportunities for wearable devices in learning to climb. In Proceedings of the 9th Nordic Conference on Human-Computer Interaction, NordiCHI '16, pp. 48:1-48:10. ACM, New York, NY, USA, 2016. doi: $10.1145 / 2971485.2971509$

[10] Y. S. Pai, M. Isogai, K. Kunze, T. Nakao, and H. Kimata. Ubitrain: Leveraging the physical and virtual environment for ubiquitous sports training. In Proceedings of the 2018 ACM International Joint Conference and 2018 International Symposium on Pervasive and Ubiquitous Computing and Wearable Computers, UbiComp '18, pp. 202-206. ACM, New York, NY, USA, 2018. doi: 10.1145/3267305.3267646

[11] J. Song, G. Sörös, F. Pece, S. R. Fanello, S. Izadi, C. Keskin, and O. Hilliges. In-air gestures around unmodified mobile devices. In Proceedings of the 27th Annual ACM Symposium on User Interface Software and Technology, UIST '14, pp. 319-329. ACM, New York, NY, USA, 2014. doi: 10.1145/2642918.2647373

[12] Y. Tanaka, H. Kon, and H. Koike. A real-time golf-swing training system using sonification and sound image localization. In Proceedings of the 24th ACM Symposium on Virtual Reality Software and Technology, VRST '18, pp. 66:1-66:2. ACM, New York, NY, USA, 2018. doi: 10. $1145 / 3281505.3281604$

[13] C. Zhang, F. Yang, G. Li, Q. Zhai, Y. Jiang, and D. Xuan. Mv-sports: A motion and vision sensor integration-based sports analysis system. In IEEE INFOCOM 2018 - IEEE Conference on Computer Communications, pp. 1070-1078, April 2018. doi: 10.1109/INFOCOM.2018. 8485910

[14] B. Zhou, H. Koerger, M. Wirth, C. Zwick, C. Martindale, H. Cruz, B. Eskofier, and P. Lukowicz. Smart soccer shoe: Monitoring football interaction with shoe integrated textile pressure sensor matrix. In Proceedings of the 2016 ACM International Symposium on Wearable Computers, ISWC '16, pp. 64-71. ACM, New York, NY, USA, 2016. doi: $10.1145 / 2971763.2971784$

${ }^{5}$ https://treality.com/ 